\title{
Actin Cytoskeleton and Membrane Interactions: Role in GPCR Function and Organization
}

\author{
SHIKHA PRAKASH, ANJALI KRISHNA and DURBA SENGUPTA* \\ CSIR-National Chemical Laboratory, Dr. Homi Bhabha Road, Pune 411 008, India
}

(Received on 21 March 2018; Accepted on 15 September 2018)

\begin{abstract}
Cell signaling networks are generally initiated at the cell membrane and mediated by receptors such as the G proteincoupled receptors (GPCRs). Adjacent to the cell membrane lies a complex network of proteins - the actin cytoskeleton to which several structural and physiological roles have been attributed. An emerging role of the cytoskeleton is to modulate GPCR function and organization, either directly or indirectly. The GPCR-cytoskeleton cross-talk is a complex hierarchical process where each step has its own set of rules and combinations. Due to the inherent complexity involved at each step and the multiple spatio-temporal levels, a complete picture is yet to emerge. In this review article, we provide an overview of actin-membrane interactions and how they modulate GPCR function and organization. In this context, we briefly discuss the structural characterization of actin monomers and filaments together with their interactions with partner proteins, such as the actin binding proteins. The effect of actin interactions on membrane domains is examined that assumes significance in light of membrane modulated effects in GPCR function. We aim to bring together concepts in GPCR signaling and cytoskeletal dynamics towards addressing emerging concepts in GPCR function.
\end{abstract}

Keywords: Actin; Cytoskeleton; GPCR; Cell-membrane; Pinning Sites; ABPs

\section{Introduction}

Cell signaling is the process by which cells relay specific information from the environment to the interior by sensing external cues (Alberts et al., 2013; Hancock, 2016). It encompasses pathways mediated by a large number of proteins that interact to form communication networks which extend from the membrane to the nucleus. Recent reports suggest that signal transduction is sensitively dependent on how these molecules are organized and interact collectively. Previous biochemical and physiological studies have identified several players, such as membrane-bound $\mathrm{G}$ protein-coupled receptors (GPCRs), intracellular GTP-binding proteins and protein kinases. Several signaling pathways have also been identified, a few of which converge on proteins that directly regulate the behavior and organization of the actin cytoskeleton in the cytoplasm (Alberts et al., 2013; Hancock, 2016). The lacuna in our understanding lies in the molecular basis of these cascades, the cross-talk between the players and how the cytoskeleton could in return modulate the function of the receptors that initiate the signal. In this review, we discuss the role of the cytoskeleton in modulating GPCR function and organization. Overall, we observe several points of cross-talk between actin and GPCRs. Reports on direct interactions between GPCRs and the cytoskeleton are limited, but evidence pointing to indirect interactions through actin regulating proteins and the membrane is increasing. We briefly overview structural dynamics of GPCRs and the cytoskeleton, and focus mainly on reports of the interplay between the cytoskeleton and cell membrane embedded GPCRs.

\section{Structural and Functional Characteristics of GPCRs}

The GPCR family of proteins are important seven transmembrane receptors (7TM receptors) that perceive extracellular signals and in response propagate a cellular signaling cascade (Pierce et al., 2002; Dohlmn, 2015; Thal et al., 2018). They are the

*AuthorforCorrespondence: E-mail: d.sengupta@ncl.res.in; s.prakash@ncl.res.in 
largest and most diverse class of eukaryotic membrane proteins. The GPCR family includes receptors involved in the recognition of light, taste, odors, hormone, pain, neurotransmitters and several other stimuli (Rosenbaum et al., 2009; Hilger et al., 2018). Given the implications of GPCRs in disease and physiology, they form attractive targets for approximately one third of the currently marketed drugs (Campbell and Smrcka, 2018; Hauser et al., 2017; Sid, 2018; Sriram and Insel, 2018).

Advances in crystallography and more recently the advent of cryo-electron microscopy (cryo-EM) has helped uncover several facets of the structure and activation of monomeric GPCRs (Granier and Kobilka, 2012; Boland et al., 2017; Venkatakrishnan et al., 2013). A representative member of the GPCR family, the serotonin $_{1 \mathrm{~A}}$ receptor (Paila et al., 2011; Patra et al., 2015) is shown in Fig. 1. It is an important neurotransmitter receptor that has a role in cognitive, behavioral, and developmental functions (Kalipatnapu and Chattopadhyay, 2007; Nautiyal and Hena, 2017). Archetype GPCR signaling involves activation by extracellular ligands (such as serotonin) (Saxena and Chattopadhyay, 2011; Pucadyil and Chattopadhyay, 2004; Wirth et al., 2017) and coupling of transducers such as $\mathrm{G}$ protein on the intracellular site (Wirth et al., 2017; Thal et al., 2018). Over time evidence has accumulated that GPCRs not only signal through heterotrimeric $\mathrm{G}$ proteins, but also via arrestins and other G-protein-independent pathways (Hilger et al.,

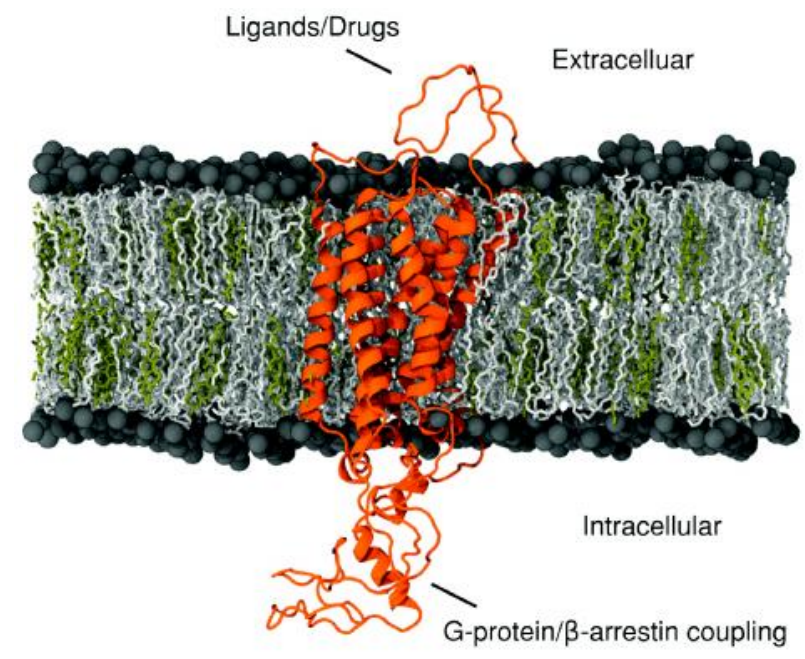

Fig. 1: Structural representation of a representative GPCR, the serotonin ${ }_{1 \mathrm{~A}}$ receptor. The receptor is represented in orange, lipids in gray and cholesterol in olive green color
2018; Latorraca et al., 2017; Dwivedi et al., 2018; Wisler et al., 2018). Ligand mediated GPCR activation is dynamic in nature, resulting in concerted conformational changes in the transmembrane domains, and facilitating downstream protein interactions (Hilger et al., 2018; Dror et al., 2011; Nygaard et al., 2013; Gether, 2000; Manglik and Kruse, 2017). We direct readers to recent reviews for an overview of structural details and activation mechanisms (Weis and Kobilka, 2018; Erlandson et al., 2018; Latorraca et al., 2017).

An emerging concept in the field of GPCR biology is the role of its environment and other proteins in the regulation of GPCR function (Chattopadhyay, 2014). Several modulators of GPCR function have been identified, such as a wide repertoire of lipids, especially cholesterol (Jafurulla and Chattopadhyay, 2013; Oates and Watts, 2011; Gonzalez et al., 2017; Gimpl and Gehrig-Burger, 2012 ). It has been shown that tuning the membrane composition can alter GPCR function and organization. Membrane effects can be specific as well as non-specific in nature (Prasanna et al., 2014; Paila and Chattopadhyay, 2009; Jaipuria et al., 2018; Periole, 2016). We direct the readers to reviews (Oates and Watts, 2011; Chattopadhyay, 2014; Sengupta and Chattopadhyay, 2015; Sengupta et al., 2018) for a comprehensive insight into lipid effects on GPCR function. Furthermore, the association of GPCRs to form dimers and higher order oligomers has been reported (Chakraborty and Chattopadhyay, 2014) and its functional significance continues to be debated. The organization of GPCRs into varying membrane nano domains and oligomers appears to directly modulate GPCR function (Conn, 2013). However, accumulating evidence suggests that the actin cytoskeleton and GPCR function can be related either directly or indirectly (Vazquez-Victorio et al., 2016; Haanappel and Salome, 2017). In addition, actin has been reported to regulate related cell signaling pathways through compartmentalization, dynamics and clustering (Mattila et al., 2016; Krapf , 2018; Köster et al. 2016). Thus, the actin cytoskeleton could prove to be the missing piece of the GPCR jigsaw puzzle.

\section{Actin: The Hidden Player}

A schematic representation of membrane-embedded GPCRs along with the underlying cytoskeletal network is shown in Fig. 2. A priori there appear to be no 


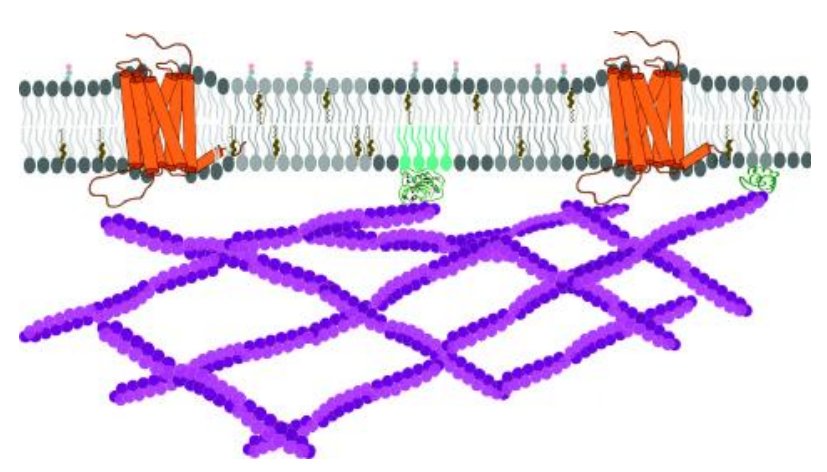

Fig. 2: A schematic representation of membranecytoskeleton interactions. The lipid bilayer is shown in gray with gradients to highlight different lipid types. Cholesterol is represented in tan and PIP2 in cyan. The GPCRs have been highlighted in orange, ABPs in green and the underlying actin cytoskeleton in shades of purple

direct links between the GPCR and the actin cytoskeleton. However, Kusumi and co-workers showed that actin influences opioid receptor diffusion using single-molecule techniques (Suzuki et al., 2005). Furthermore, a link between signaling and receptor mobility was established in the serotonin ${ }_{1 \mathrm{~A}}$ receptor, thereby implying that actin could play a regulatory role in GPCR signaling (Ganguly et al., 2008). Using quantitative fluorescence correlation spectroscopy (FCS), Chattopadhyay and co-workers demonstrated that the serotonin $_{1 \mathrm{~A}}$ receptor is confined to cell membranes, possibly due to its interaction with the actin cytoskeleton (Ganguly and Chattopadhyay, 2010). Interestingly, the reorganization of the actin cytoskeleton upon GPCR signaling has also been reported (Ganguly, Saxena and Chattopadhyay, 2011). Taken together, the data suggests a dual role of actin in modulating GPCR organization as well as function, although the molecular details remains unclear. We review below the structural dynamics of actin, its interactions with the membrane and the crosstalk with GPCRs.

\section{Structural Dynamics of The Actin Cytoskeleton}

The actin cytoskeleton is a complex meshwork of proteins extending from the cell membrane to the nucleus that plays an important role in cell shape and motility. Actin filaments are enriched in a narrow region underlying the plasma membrane known as the cortex, where they are organized into a network. Actin occurs in two different forms: monomeric globular actin (G- actin) and polymeric filamentous actin (F-actin). Gactin has been well characterized structurally and several crystal structures are available (Mouilleron et al., 2012 ; Kotila et al., 2018). Over the years, there has been a gradual increase in both crystal (Holmes et al., 1990; Oda et al., 2009; von der Ecken et al., 2014) and cryo-EM (Fujii et al., 2010; Galkin et al., 2010, 2015) structural data on F-actin with increasing resolution (Oosawa, 2018). Quite recently, the cryo-EM structures of an actin filament in complex with myosin VI (Gurel et al., 2017) and cofilin (Tanaka et al., 2018) were resolved. For a direct comparison, we have represented the two forms of actin in Fig. 3. It can be discerned from the figure that F-actin is composed of two parallel strands of actin monomers, in which each G-actin protomer rotates $166^{\circ}$ to give a double stranded helix appearance. Several structural changes are associated with the inter conversion of monomeric G-actin to filamentous F-actin, including a "flattening" of the protomers. This is achieved by a relative rotation of two of the major domains of Gactin by $20^{\circ}$ in a propeller-like (or swing door) manner (Kudryashov and Reisler, 2013). This inter conversion

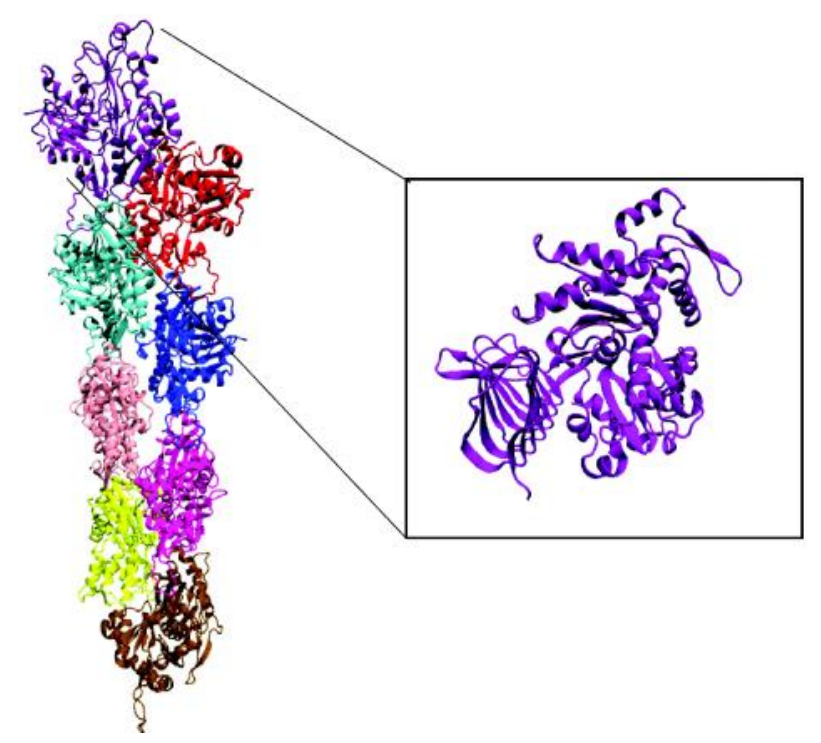

Fig. 3: The structural representation of (inset) actin monomer and (left) actin filament. The actin protomers are shown in cartoon representation and colored individually. The PDB structure of actin monomer (PDB ID: 6FM2) corresponds to the recently deposited crystal structure ADP-G-actin in com- plex with CAP (not shown) (Kotila et al., 2018). The actin filament structure corresponds to the structure determined by cryo EM discussed above (PDB ID: 6BNO) (Gurel et al., 2017) 
is an active, cyclic process involving polymerization and de-polymerization, although factors governing $\mathrm{G}$ to $\mathrm{F}$ actin transition are still being explored (Oda et al.,2009; Pfaendtner et al., 2010; von der Ecken et al., 2014; Galkin et al., 2015; von der Ecken and Heissler, 2016). The details of these actin filament networks and the molecular mechanisms controlling actin filament dynamics have been reviewed previously (Pollard et al., 2000; Pollard, 2016).

\section{Interactions of The Actin Cytoskeleton with Cell Membranes}

The earliest evidence on actin-membrane interactions was reported several from several biochemical studies (Branton et al., 1981; Luna and Hitt, 1992; Hitt and Luna, 1994; Cowin and Burke, 1996). In general, actin interacts with the membrane mainly through indirect interactions, although direct interactions have also been reported (Sasaki et al., 2014; Ashdown and Burn, 2017). Indirect interactions occur through cytoskeletal elements linked to transmembrane proteins or actin binding proteins (ABPs) that interact with the inner leaflet of the plasma membrane or organelle membrane (Senju and Lappalainen, 2019; Lemiere $e t$ al., 2016; Fritzsche et al., 2017; Papadopulos, 2017). Further details on ABPs is given in the next section. Membrane phospholipids have been shown to modulate the "pinning" of the actin cytoskeleton. Membrane phosphoinositides constitute less than 5\% of the membrane lipids, but are able to modulate attachment sites of membrane-actin networks (Liu and Fletcher, 2006; Senju and Lappalainen, 2019). Recently it was shown that although different ABPs interact with membranes through distinct domains, they bind phosphoinositide rich membranes via similar multivalent electrostatic interactions (Senju et al., 2017).

\section{Actin-binding Proteins (ABPs)}

The role of ABPs in the organization and dynamics of the actin cytoskeleton has been well characterized (Lappalainen, 2016). They perform several roles, such as catalyzing nucleotide exchange in actin monomers, and initiation, elongation, termination and cross-linking of actin filaments (Uribe and Jay, 2007; Pollard, 2016; Tanaka et al., 2018). Cofilin is the main player involved in the depolymerization of actin filaments. It does so by preferentially binding to ADP-actin and subsequently sequesters resulting monomers in the
ADP-bound form, thus preventing their incorporation into filaments (Pollard, 2016). It controls the contractibility and motility of various cellular and subcellular components. Recently, a high resolution cryoEM structure of cofilin bound to actin (cofilactin) was resolved by Tanaka and co-workers (Tanaka et al., 2018). The cofilin-actin interface could be delineated and the authors proposed models for the cooperative binding of cofilin to actin (Tanaka et al., 2018). Another ABP, profilin, can reverse the effect of cofilin, thus allowing the incorporation of actin monomers into filaments. Other important ABPs include Thymosinb4, Gelsolin, Arp2/3 complex, Filamin, Dystrophin and we direct the readers to more extensive reviews on ABPs (Dos Remedios et al., 2003; Winder and Ayscough, 2005; Uribeand Jay, 2007; Kristo et al., 2016; Pollard, 2016).

ABPs have been reported to act as (virtual) bridges by simultaneously interacting with the membrane and the cytoskeleton, such as annexins (Winder and Ayscough, 2005). Proteins that link the actin cytoskeleton to membranes or membrane proteins include dystrophin, utrophin, talin and vinculin (Winder and Ayscough, 2005). The binding of $\mathrm{PI}(4,5) \mathrm{P} 2$ via Arp2/3 initiates actin polymerization which is promoted by actin nucleation factors, WAVE and WASP. On the other hand the F-actin severing protein $\mathrm{ADF} / \mathrm{Cofilin}$, and the $\mathrm{G}$-actin binding protein profilin, actin-capping protein, are all constrained by binding PI(4,5)P2 (Bezanilla et al., 2015).

\section{Effect of Cytoskeletal Pinning Sites on Membrane Organization}

The attachment of actin networks to the membrane at the "pinning sites" has been reported to lead to membrane reorganization (Koster et al., 2016; Lemiere et al., 2016; Fritzsche et al., 2017; Papadopulos, 2017). For instance, actin can modulate the formation and size of membrane microdomains which have been proposed to form scaffolds that compartmentalize various signal transducers (Kusumi et al., 2011). In addition, it was been shown that the pinning sites could give rise to confined diffusion at the membrane (Kusumi et al., 2012). Monte Carlo (MC) simulations of lipid membranes have demonstrated that both phase separation and lateral diffusion in membranes can be strongly affected by actin (Ehrig et al., 2011; Machta et al., 2011). In fact, 
several membrane proteins and lipids exhibit restricted diffusion along the membrane, spanning over micrometer and nanometer length-scales and microsecond and nanosecond time-scales (Kusumi et al., 2012; Li et al., 2018; Cebecauer et al., 2018; Kusumi and Suzuki, 2005; Fujiwara et al., 2016; Kusumi et al., 2011).

Actin may have opposing effects of organization and phase mixing in membranes (Arumugam et al., 2015). Using bacterial cytoskeletal, FtsZ/MinD system, together with giant unilamellar vesicles, it was demonstrated that the artificial membrane-associated cytoskeleton, on the one hand, suppresses large scale phase separation below the phase transition temperature, and, on the other hand, preserves phase separation above the transition temperature (Arumugam et al., 2015). Using a combined experimental and modeling approach, it was proposed that membrane composition, the membrane curvature, and the actin pinning sites are coupled (Honigmann et al., 2014). An important point to note here is that the membrane demixing was obtained even in the presence of simple pinning sites via biotinylated lipid streptavidin complexes (Honigmann et al., 2014). Interestingly, when the membrane-cytoskeleton interaction is mediated by proteins that preferentially partition into a specific domain type, cytoskeleton meshwork organization and lipid domain distribution are intimately related. Pinning of a specific phase by the preference of pinning molecules has been qualitatively shown in vitro (Zhao et al., 2013). Taken together, these studies indicate that actin-membrane interactions could involve a wide range of mechanisms and general principles are emerging despite the lack of a detailed molecular picture.

\section{Cross-talk Between Actin and GPCRs}

Despite the first reports on cytoskeleton mediated GPCR diffusion and mobility (Suzuki et al., 2005; Ganguly et al., 2008), the precise interactions between actin and membrane proteins remain unclear. This is probably due to the spatio-temporal dynamics of both GPCRs and actin and the inherent limitations of analyzing membrane-bound dynamics. Several strategies have been employed to examine the binding of actin to membrane channel proteins, including coimmuno precipitation, surface plasmon resonance, fluorescence resonance energy transfer (FRET), and atomic force microscopy (Sheng and Pak, 2000). Direct interactions between ion channels and actin cytoskeleton has been reported (Sasaki et al., 2014), and could be relevant for GPCR interactions as well. However, no direct actin-GPCR structure modules have been observed so far.

There are limited reports on the direct interactions between ABPs and GPCRs. $\alpha$-actinins ( $\alpha$-actinin- 1 to $\alpha$-actinin- 4 ) are a class of ABPs that not only bind to actin filaments but also modulate the activity of membrane receptors (Foley and Young, 2014). A recent study by Sun et al. (Sun et al., 2016) showed that the binding of the adenosine $\mathrm{A}_{2}$ receptor with actinin-1 is destabilized in the presence of two point mutations. Bezanilla and co-workers (Bezanilla et al., 2015) characterized the interaction of $\alpha$-actinin4 with group1 metabotropic glutamate receptors (mGluR) which in turn governs remodeling of dendritic spines in primary neurons. Similarly, the actin-binding protein 280 (ABP-280) has been shown to associate with dopamine D3 receptors (Li et al., 2002). Interestingly, a conserved filamin binding sequence was found in the extracellular domains of $>20 \%$ of the 824 GPCRs encoded in the human genome through an exhaustive sequence-based analysis (Tirupula et $a l ., 2015)$. Direct binding between Filamin-A and select GPCRs was confirmed through biochemical experiments (Tirupula et al., 2015).

\section{GPCR Organization is Modulated by the Cytoskeleton}

Reports suggest that actin modulates GPCR organization in at least three possible (interconnected) ways: confining (corraling) diffusion, altering the oligomerization state and modulating association kinetics. Mechanisms underlying corralled diffusion are discussed above (Suzuki et al., 2005; Ganguly et al., 2008). Such confined or corralled diffusion could have functional consequences (Ganguly et al., 2008).

To investigate the role of actin cytoskeleton in GPCR oligomer size, Chattopadhyay and co-workers utilized homo-FRET approaches in live cells and demonstrated that the actin cytoskeleton could directly modulate the organization of the receptor (Ganguly, Clayton and Chattopadhyay, 2011). The result suggest that destabilization of the actin cytoskeleton by cytochalasin $\mathrm{D}$ lead to an increased contribution from higher order oligomers in live cells (Ganguly, Clayton 
and Chattopadhyay, 2011). Interestingly, a cell typedependent clustering of $\beta_{2}$-adrenergic receptor was reported using super resolution microscopy approaches (Scarselli et al., 2012). It was shown that the cluster size depended on actin cytoskeleton integrity but not on membrane microdomains (Scarselli et al., 2012). In addition, the cluster size and dynamics of the chemokine receptor CXCR4 were reported to be directly modulated by the actin cytoskeleton (Martiýnez-Munoz et al., 2018). Interestingly, the effect of actin on GPCR oligomer/cluster size is receptor dependent. GABA-B oligomers were reported to directly interact with the actin cytoskeleton and form large ordered clusters Calebiroa et al. (2013). However, disruption of actin filaments lead to reduced cluster sizes in $\beta_{2}$-adrenergic receptor, but not in GABA-B complexes (Scarselli et al., 2016; Calebiroa et al., 2013). It has been suggested that the actin cytoskeleton could help to confine specific GPCRs to specific domains in order to increase efficiency of them encountering their signaling partners (Scarselli et al., 2016). These results imply that the actin cytoskeleton could play a regulatory role in GPCR organization.

Evidence on the altered GPCR association kinetics by actin cytoskeleton comes from mathematical modeling and comparison to single particle tracking measurements. We have previously developed a kinetic Monte Carlo model in which the membrane was represented by a two-dimensional lattice with periodic boundary conditions and the receptors as point particles (Deshpande et al., 2017). As shown in Fig. 4, the square lattice was initially randomly populated with a given density of receptors. Each receptor was propagated with displacement steps $\delta_{\text {ri }}$, derived from the expression of the mean squared displacement of a two-dimensional random walk, by the relation: $\delta r_{i}=\sqrt{ }\left(4 D_{i} \delta t\right)$ where, $i=1,2$ indicates whether the particle is a monomer or dimer, $D_{i}$ is the diffusion coefficient of the ith particle and $\delta t$ the time interval (Pawar et al., 2015; Athale et al., 2014). In the model, the monomers were allowed to associate at adjacent sites with the probability of association $\left(\mathrm{P}_{\text {on }}\right)$ given by $\mathrm{P}_{\text {on }} \approx \mathrm{k}_{\text {on }} \delta \mathrm{t}$, where $\mathrm{k}_{\text {on }}$ is the on-rate. Dimer dissociation was based on the probability $\mathrm{P}_{\text {off }} \approx \mathrm{k}_{\text {off }} \delta \mathrm{t}$, where $\mathrm{k}_{\text {off }}$ is the off rate. Cytoskeletal filaments were modeled as barriers for receptor diffusion, with a hopping probability, $\mathrm{P}_{\text {hop' }}$, similar to the 'picket fence' or corralled diffusion

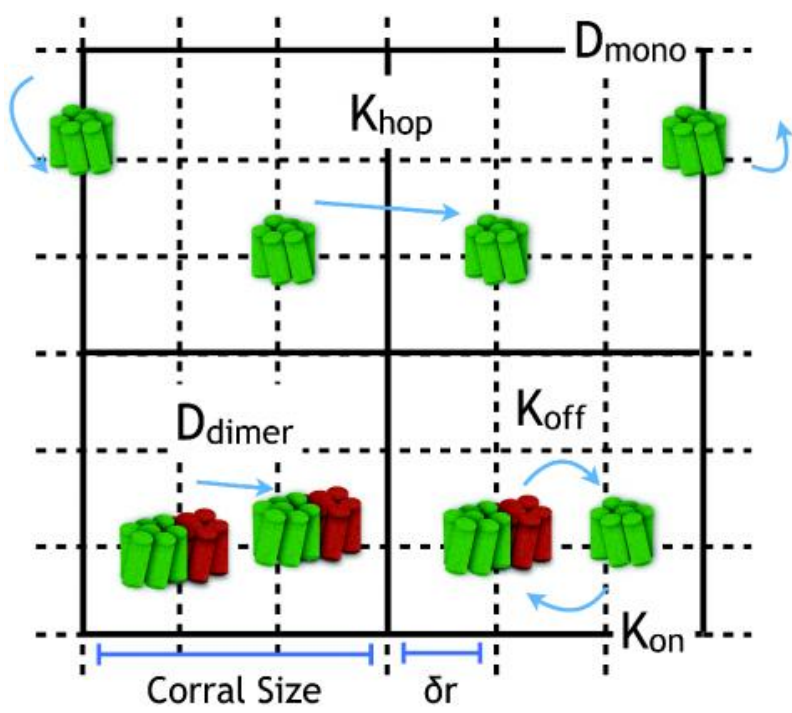

Fig. 4: Schematic representation of the kinetic Monte Carlo model of the classical association-diffusion model. A two-dimensional lattice is defined with periodic boundary conditions to represent the membrane. Receptors are colored in green and orange. The steps taken in the consecutive steps are marked. Cytoskeleton corrals are represented as underlying barriers

model (Suzuki et al., 2005). The model predicts a continuous change in the percentage of receptor dimers, with increasing corral size as well as probability of hopping. The monomer-dimer equilibrium of the GPCRs predicted are in line with previous experimental findings (Kasai et al., 2011; Calebiroa et al., 2013). The model reveals a complex interplay between cytoskeletal components and their influence on receptor association kinetics within the features of the membrane landscape. The results suggest a functional coupling between receptor and actin and a modulation of the dynamics of receptor organization.

\section{Cytoskeleton Mediated Trafficking of GPCRs}

Membrane trafficking plays a critical role in regulating cell signaling, including downstream responses mediated by GPCRs. They are sorted and internalized via different endocytotic pathways to be either degraded or recycled (Pons et al., 2017). Actin filaments are important players in receptor sorting within endosomes, aided by proteins such as WASH and the Arp 2/3 complex (Derivery et al., 2012; Gomez and Billadeau, 2009) and specialized protein interactions. A recent study has shown that phosphorylation of Filamin A (FLNa) governs 
chemokine receptor CCR2B recycling through endocytosis (Pons et al., 2017). Alternatively, actin binding sequences may be present in GPCRs like the $\beta_{2}$-adrenergic receptor that sequester the receptor in endosomes comprising actin (Puthenveedu et al., 2010). This in turn is mediated by the actin-sorting nexin 27-retromer tubule (ASRT) machinery and PDZ proteins that bind to the cortical actin cytoskeleton prior to the recycling of the receptor (Bowman et al., 2016; Varandas et al., 2016; Bahouth and Nooh, 2017). Similarly, a conserved filamin binding sequence was found across the cytoplasmic domains of several GPCRs, and their direct binding to filamin-A was reported (Tirupula et al., 2015). Recent evidence suggests that GPCR signaling can persist in the endosomes (Chaturvedi et al., 2018; Irannejad and von Zastrow, 2014), and cytoskeletal coupling could be an important regulatory principle in this context.

\section{Conclusions and Future Perspectives}

The understanding of actin cytoskeleton networks and their role in GPCRs signaling has undergone significant advancement due to improved experimental techniques with increased spatio-temporal resolution. It now appears that actin serves as a critical point of integration of receptor signaling such that changes in the cytoskeleton induced by one signal can readily influence the function of other receptors. Ligand

\section{References}

Alberts B, Bray D, Hopkin K, Johnson A, Lewis J, Raff M, Roberts K and Walter P (2013), Essential cell biology, Garland Science

Arumugam S, Eugene, P P and Schwille P (2015) Cytoskeletal pinning controls phase separation in multicomponent lipid membranes Biophys J 108 1104-1113

Ashdown G W and Burn G L (2017) Live-cell super-resolution reveals $\mathrm{F}$-actin and plasma membrane dynamics at the $\mathrm{T}$ cell synapse Biophys J 112 1703-1713

Athale C A, Dinarina A, Nedelec F and Karsenti E (2014) Collective behavior of minus-ended motors in mitotic microtubule asters gliding toward dna Phys Biol 11016008

Bahouth S W and Nooh M M (2017) Barcoding of GPCR trafficking and signaling through the various trafficking roadmaps by compartmentalized signaling networks Cell Signal 36 42-55 binding as well as G protein coupling in GPCRs has been shown to be altered by indirect changes in physico-chemical properties space of the membrane (Prasad et al., 2009; Pal et al., 2016). Additionally, modulation of the GPCR association has been reported to be directly linked to indirect effects (Prasanna et al., 2015; Pawar, Prasanna and Sengupta, 2015). Factin network assembly, organization, and dynamics are controlled by the spatial and temporal regulation of the activity of ABPs that in turn could affect membrane dynamics and properties. Consequently, actin can indirectly affect GPCRs by altering the physical properties of membrane. In this article, we have highlighted representative experimental and computational approaches to address these questions in GPCR biophysics. These are exciting times for actin cytoskeleton researchers since we are able to use complimentary approaches to address interactions problems at a spatio-temporal resolution that was not possible to achieve even a few years back. Unraveling the molecular basis of GPCR-actin will be an important step towards understanding human health and disease.

\section{Acknowledgement}

The authors thank Prof. Amitabha Chattopadhyay and Dr. Xavier Prasanna for discussions. DS gratefully acknowledges the Dept. Science Technology (DST, (Govt. of India) grant: EMR/2016/002294) for funding.

Bezanilla M, Gladfelter A S, Kovar D R and Lee W L (2015) Cytoskeletal dynamics: A view from the membrane $J$ Cell Biol 209 329-337

Boland A, Chang L and Barford D (2017) The potential of cryoelectron microscopy for structure-based drug design Essays Biochem 61 543-560

Bowman S L, Shiwarski D J and Puthenveedu M A (2016) Distinct $\mathrm{G}$ protein-coupled receptor recycling pathways allow spatial control of downstream $G$ protein signaling $J$ Cell Biol 214 797-806

Branton D, Cohen C M and Tyler J (1981) Interaction of cytoskeletal proteins on the human erythrocyte membrane Cell 24 24-32

Calebiroa D, Riekena F, Wagner J, Sungkaworna T, Zabela U, Borzid A, Cocuccie E, Zürna A and Lohse M J (2013) Single-molecule analysis of fluorescently labeled g-proteincoupled receptors reveals complexes with distinct 
dynamics and organization Proc Nat Acad Sci 110 743748

Campbell A P and Smrcka A V (2018), Targeting G proteincoupled receptor signaling by blocking $\mathrm{G}$ proteins Nature 17 789-803

Cebecauer M, Amaro M, Jurkiewicz P, Sarmento M J, Sachi R, Cwiklik L and $\mathrm{H}$ of $\mathrm{M}$ (2018) Membrane lipid nanodomains Chem Rev 118 11259-11297

Chakraborty H and Chattopadhyay A (2014) Excitements and challenges in GPCR oligomerization: Molecular insight from FRET ACS Chem Neurosc 6 199-206

Chattopadhyay A (2014) GPCRs: Lipid-dependent membrane receptors that act as drug targets Adv in Biol 143023

Chaturvedi M, Schilling J, Beautrait A, Bouvier M, Benovic J L and Shukla A K (2018) Emerging paradigm of intracellular targeting of GPCRs Tr Biochem Sci 43 533-546

Conn P M (2013) G Protein Coupled Receptors: Trafficking and Oligomerization Methods in enzymology, Elsevier Science $52169-90$

Cowin P and Burke B (1996) Cytoskeleton-membrane interactions Curr Opin Cell Biol 8 56-65

Derivery E, Helfer E, Henriot V and Gautreau A (2012) Actin polymerization controls the organization of WASH domains at the surface of endosomes PLoS One 7 e39774

Deshpande S, Pawar A, Dighe A, Athale C and Sengupta D (2017) Role of spatial inhomogenity in GPCR dimerisation predicted by receptor association-diffusion models, Phy Biol 14036002

Dohlmn H G (2015) Thematic minireview series: Cell biology of G protein signaling J Biol Chem $2906679-6670$

Dos Remedios C G, Chhabra D, Kekic M, Dedova I V, Tsubakihara M, Berry D A and Nosworthy N J (2003) Actin Binding Proteins: Regulation of Cytoskeletal Microfilaments Physiol Rev 83 433-473

Dror R O, Pan, AC, Arlow D H, Borhani D W, Maragakis P, Shan Y, Xu H and Shaw D E (2011) Pathway and mechanism of drug binding to G-protein-coupled receptors Proc Natl Acad Sci 108 13118-13123

Dwivedi H, Baidya M and Shukla A K (2018) GPCR signaling: The interplay of Gai and $\beta$-arrestin Curr Biol 28 324-327

Ehrig J, Petrov E P and Schwille P (2011) Near-critical fluctuations and cytoskeleton-assisted phase separation lead to sub diffusion in cell membranes Biophys $J \mathbf{1 0 0}$ 80-89

Erlandson S C, McMahon C and Kruse A C (2018) Structural basis for $\mathrm{G}$ protein-coupled receptor signaling Ann Revi Biophys 47 1-18

Foley K S and Young P W (2014) The non-muscle functions of actinins: An update Biochem J 459 1-13

Fritzsche M, Li D, Colin-York H, Chang V T, Moeendarbary E, Felce J H, Sezgin E, Charras G, Betzig E and Eggeling C (2017) Self-organizing actin patterns shape membrane architecture but not cell mechanics Nat Commun 814347

Fujii T, Iwane A H, Yanagida T and Namba K (2010) Direct visualization of secondary structures of F-actin by electron cryomicroscopy Nature $\mathbf{4 6 7} 724$

Fujiwara T, Iwasawa K, Kalay Z, Tsunoyama T, Watanabe Y, Umemura Y, Murakoshi H, Suzuki K, Nemoto Y, Morone $\mathrm{N}$ and Kusumi A (2016) Confined diffusion of transmembrane proteins and lipids induced by the same actin meshwork lining the plasma membrane Mol Biol Cell 27 1101-1120

Galkin V E, Orlova A, Matthijn R J V, Schr"and Egelman E H (2015) Near-atomic Resolution for Oner State F-Actin 23 173-182

Galkin V E, Orlova A, Schr"der G F and Egelman E H (2010) Structural polymorphism in F-actin Nat Struct Mol Biol 17 1318-1323

Ganguly S and Chattopadhyay A (2010) Cholesterol depletion mimics the effect of cytoskeletal destabilization on membrane dynamics of the serotonin ${ }_{1 \mathrm{~A}}$ receptor: A zFCS study Biophys J 99 1397-1407

Ganguly S, Clayton A H A and Chattopadhyay A (2011) Organization of higher-order oligomers of the serotonin ${ }_{1 \mathrm{~A}}$ receptor explored utilizing homo-FRET in live cells Biophys J 100 361-368

Ganguly S, Pucadyil T J and Chattopadhyay A (2008) Actin cytoskeleton-dependent dynamics of the human serotonin $_{1 \mathrm{~A}}$ receptor correlates with receptor signaling Biophys J 95 451-463

Ganguly S, Saxena R and Chattopadhyay A (2011) Reorganization of the actin cytoskeleton upon G-protein coupled receptor signaling Biochim Biophys Acta 1808 1921-1929

Gether U (2000) Uncovering molecular mechanisms involved in activation of G protein-coupled receptors Endocr Rev 21 90-113

Gimpl G and Gehrig-Burger K (2012) Specific and Non specific Regulation of GPCR Function by Cholesterol, John Wiley and Sons, Inc., pp. 205-230

Gomez T S and Billadeau D D (2009) A FAM21-containing WASH complex regulates retromer-dependent sorting $D e v$ Cell 17 699-711

Gonzalez R G, Albasanz J L, Espigares I R, Pastor M, Sanz F, Solano M M, Manna, M, Seara H M, Hildebrand P W, Martn M and Selent J (2017) Membrane cholesterol access 
into a G-protein-coupled receptor Nature $\mathbf{8} 14505$

Granier S and Kobilka B (2012) A new era of GPCR structural and chemical biology Nat Chem Biol 8 670-673

Gurel P S, Kim L Y, Ruijgrok P V, Omabegho T, Bryant Z and Alushin G M (2017) Cryo-EM structures reveal specialization at the myosin VI-actin interface and a mechanism of force sensitivity Elife 6 e 31125

Haanappel E and Salome L (2017) G-protein-coupled receptors: Membrane diffusion and organization matter, Membrane Org Dynamics 243-258

Hancock J T (2016) Cell Signaling, Oxford University Press

Hauser A S, Chavali S, Masuho I, Leonie J, Martemyanov K A, Gloriam, D E and Babu M M (2017), Pharmacogenomics of GPCR Drug Targets Cell 172 41-54

Hilger D, Masureel M and Kobilka B K (2018) Structure and dynamics of GPCR signaling complexes Nat Struct Mol Biol 25 4-12

Hitt A L and Luna E J (1994) Membrane interactions with the actin cytoskeleton Curr Opin Cell Biol 6 120-130

Holmes K C, Popp D, Gebhard W and Kabsch W (1990) Atomic model of the actin filament Nature $\mathbf{3 4 7} 44$

Honigmann A, Sadeghi S, Keller J, Hell S W, Eggeling C and Vink R (2014) A lipid bound actin meshwork organizes liquid phase separation in model membranes Elife 3 e01671

Irannejad R and von Zastrow M (2014) GPCR signaling along the endocytic pathway Curr Opin Cell Biol 27 109-116

Jafurulla M and Chattopadhyay A (2013) Membrane lipids in the function of serotonin and adrenergic receptors Curr Med Chem 20 47-55

Jaipuria G, Ukmar-Godec T and Zweckstetter M (2018) Challenges and approaches to understand cholesterolbinding impact on membrane protein function: an NMR view Cell Mol Life Sci 75 2137-2151

Kalipatnapu S and Chattopadhyay A (2007) Membrane organization and function of the serotonin ${ }_{1 \mathrm{~A}}$ receptor Cell Mol Neurobiol 27 1097-1116

Kasai R S, Suzuki G, Prossnitz E R, Koyama-Honda I, Nakada C, Fujiwara T K and Kusumi A (2011) Full characterization of GPCR monomer-dimer dynamic equilibrium by single molecule imaging J Cell Biol 192 463-480

Koster D V, Husain K, Iljazi, E, Bhat, A, Bieling P, Mullins, R D, Rao M and Mayor S (2016) Actomyosin dynamics drive loal membrane component organization in an in vitro active composite layer Proc Natl Acad Sci 113 E1645-E1654

Kotila T, Kogan K, Enkavi G, Guo S, Vattulainen I, Goode B L and Lappalainen P (2018) Structural basis of actin monomer recharging bycyclase-associated protein Nat Commun 9
$1-12$

Krapf D (2018) Compartmentalization of the plasma membrane Curr Opin Cell Biol 53 15-21

Kristo I, Bajusz I, Bajusz C, Borku'ti P and Vilmos P (2016) Actin, actin-binding proteins, and actin-related proteins in the nucleus Histochemistry and Cell Biology 145 373-388

Kudryashov D S and Reisler E (2013) ATP and ADP states Biopolymers $99245-256$

Kusumi A, Fujiwara T K, Chadda R, Xie M, Tsunoyama T A, Kalay Z, Kasai R S and Suzuki K G (2012) Dynamic organizing principles ofthe plasma membrane that regulate signal transduction: Commemorating the fortieth anniversary of singer and nicolson fluid-mosaic model Annu Rev Cell Dev Biol 28 215-250

Kusumi A and Suzuki K (2005) Toward understanding the dynamics of membrane-raft-based molecular interactions Biochim Biophys Acta 1746 234-251

Kusumi A, Suzuki K G, Kasai R S, Ritchie K and Fujiwara T K (2011) Hierarchical mesoscale domain organization of the plasma membrane Trends Biochem Sci 36 604-615

Lappalainen P (2016) Actin-binding proteins: the long road to understanding the dynamic landscape of cellular actin networks Mol Biol Cell 27 2519-2522

Latorraca N R, Venkatakrishnan A J and Dror R O (2017) GPCR dynamics: Structures in motion Chem Rev 117 139-155

Lemi'ere J, Valentino F, Campillo C and Sykes C (2016) How cellular membrane properties are affected by the actin cytoskeleton Biochimie 130 33-40

Li M, Li C, Weingarten P, Bunzow J R, Grandy D K and Zhou Q Y (2002) Association of dopamine D(3) receptors with actin-binding protein 280 (ABP-280) Biochem Pharmacol 63 859-863

Li P, Bademosi A T, Luo J and Meunier F A (2018) Actin remodeling in regulated exocytosis: Toward a mesoscopic view Trends Cell Biol 28 685-697

Liu A P and Fletcher D A (2006) Actin polymerization serves as a membrane domain switch in model lipid bilayers Biophys J 91 4064-4070

Luna E J and Hitt A L (1992) Cytoskeleton-plasma membrane interactions Science 258 955-964

Machta B B, Papanikolaou S, Sethna J P and Veatch S L (2011) Minimal model of plasma membrane heterogeneity requires coupling cortical actin to criticality Biophys J 100 16681677

Manglik A and Kruse A C (2017) Structural basis for G proteincouple receptor activation Biochem 56 5628-5634

Mart'ýnez-Mun oz L, Rodr'ýguez-Frade J M Barroso R, 
Sorzano C O’S, Torren o-Pina J A, Santiago C A, Manzo C, Lucas P, Garc'ýa-Cuesta E M, Gutierrez E et al. (2018) Separating actin-dependent chemokine receptor nanoclustering from dimerization indicates a role for clustering in cxcr4 signaling and function Mol Cell 70 106119

Mattila P K, Batista F D and Treanor B (2016) Dynamics of the actin cytoskeleton mediates receptor cross talk: An emerging concept in tuning receptor signaling J Cell Biol 212 267-280

Mouilleron S, Wiezlak M, O'Reilly N, Treisman R and McDonald N Q (2012) Structures of the Phactr1 RPEL domain and RPEL motif complexes with G-actin reveal the molecular basis for actin binding cooperativity Structure 20 19601970

Nautiyal K M and Hena R (2017) Serotonin receptors in depression: From A to B F1000 Research 6 123-135

Nygaard R, Zou Y, Dror R O, Mildorf T J, Arlow, D H, Manglik A, Pan A C, Liu C W, Fung J, Bokoch M P, Thian F S, Kobilka T S, Shaw D E, Mueller L, Prosser, R S and Kobilka B K (2013) The dynamic process of $\beta 2$-adrenergic receptor activation Cell 152 532-542

Oates $\mathrm{J}$ and Watts A (2011) Uncovering the intimate relationship between lipids, cholesterol and GPCR activation Curr Opin Struct Biol 21 802-807

Oda T, Iwasa M, Aihara, T, Ma'eda Y and Narita A (2009) The nature of the globular-to fibrous-actin transition Nature 457 441-445

Oosawa F (2018) My various thoughts on actin Biophys Physicobiol 15 151-158

Paila Y D and Chattopadhyay A (2009) 'The function of Gprotein coupled receptors and membrane cholesterol: Specific or general interaction? Glycoconj J 26 711-720

Paila Y D, Tiwari S, Sengupta D and Chattopadhyay A (2011) Molecular modelling of the human serotonin $_{1 \mathrm{~A}}$ receptor: Role of membrane cholesterol in ligand binding of the receptor Mol Biosyst 7 224-234

Pal S, Chakraborty H, Bandari S, Yahioglu G, Suhling K and Chattopadhyay A (2016) Molecular rheology of neuronal membranes explored using a molecular rotor:implications for receptor function Chem Phys Lipids 196 69-75

Papadopulos A (2017) Membrane shaping by actin and myosin during regulated exocytosis Mol Cell Neurosci 84 93-99

Patra S M, Chakraborty S, Shahane G, Prasanna X, Sengupta D, Maiti P K and Chattopadhyay A (2015) Differential dynamics of the serotonin $_{1 \mathrm{~A}}$ receptor in membrane bilayers of varying cholesterol content revealed by all atom molecular dynamics simulation Mol Membr Biol 32 127-37
Pawar A B, Prasanna X and Sengupta D (2015) Effect of lipid bilayer composition on membrane protein association $A d v$ Planar Lipid Bil Liposomes 22 43-63

Pawar A, Deshpande S, Gopal S, Wassenaar T, Athale C and Sengupta D (2015) Thermodynamic and kinetic characterization of transmembrane helix association Phys Chem Chem Phys 17 1390-1398

Periole X (2016) Interplay of $G$ protein-coupled receptors with the membrane: Insights from supra-atomic coarse grain molecular dynamics simulations Chem Rev 117 156-185

Pfaendtner J, Lyman E, Pollard, T D and Voth GA (2010) Structure and dynamics of the actin filament J Mol Biol $396252-263$

Pierce K L, Premont, R T and Lefkowitz R J (2002) Seventransmembrane receptors Nature Revs Mol Cell Biol 3 639-650

Pollard T D (2016) Actin and actin-binding proteins Cold Spring Harb Perspect Biol 8 a018226

Pollard T D, Blanchoin L and Mullins R D (2000) Molecular mechanisms controlling actin filament dynamics in nonmuscle cells Annu Rev Biophys Biomol Struct 29 545576

Pons M, Izquierdo I, Andreu-Carb’o M, Garrido G, Planagum 'a J, Muriel O, del Pozo M A, Geli M I and Aragay A M (2017) Phosphorylation of filamin A regulates chemokine receptor CCR2 recycling J Cell Sci 130 490-501

Prasad R, Singh P and Chattopadhyay A (2009) Effect of capsaicin on ligand binding activity of the hippocampal serotonin ${ }_{1 \mathrm{~A}}$ receptor Glycoconj J 26 733-738

Prasanna X, Chattopadhyay A and Sengupta D (2014) Cholesterol modulates the dimer interface of the â2-adrenergic receptor via cholesterol occupancy sites Biophys J 106 1290-1300

Prasanna X, Chattopadhyay A and Sengupta D (2015) Role of lipid-mediated effects in $\beta 2$-adrenergic receptor dimerization Adv Exp Med Biol 842 247-261

Pucadyil T J and Chattopadhyay A (2004) Cholesterol modulates ligand binding and G-protein coupling to serotonin ${ }_{1 \mathrm{~A}}$ receptors from bovine hippocampus Biochim Biophys Acta $1663188-200$

Puthenveedu M, Lauffer B, Temkin P, Vistein R, Carlton P, Thorn $\mathrm{K}$, Taunton J, Weiner O D, Parton R G and von Zastrow M (2010) Sequence-dependent sorting of recycling roteins by actin-stabilized endosomal microdomains Cell 143761 773

Rosenbaum D M, Rasmussen S G F and Kobilka B K (2009) The structure and function of G-protein-coupled receptors Nature 459 356-363

Sasaki S Yui N and Noda Y (2014) Actin directly interacts with 
different membrane channel proteins and influences channel activities: AQP2 as a model Biochim Biophys Acta 1838 514-520

Saxena R and Chattopadhyay A (2011) Membrane organization and dynamics of the serotonin ${ }_{1 \mathrm{~A}}$ receptor in live cells $J$ Neurochem 116 726-733

Scarselli M, Annibale P, McCormick P J, Kolachalam S, Aringhieri S, Radenovic A, Corsini G U and Maggio R (2016) Revealing G-protein-coupled receptor oligomerization at the single-molecule level through a nanoscopic lens: methods, dynamics and biological function FEBS $J \mathbf{2 8 3}$ 1197-1217

Scarselli M, Annibale P and Radenovic A (2012) Cell type-specific $\beta 2$-adrenergic receptor clusters identified using photoactivated localization microscopy are not lipid raft related, but depend on actin cytoskeleton integrity $\mathrm{J}$ Biol Chem 287 16768-16780

Sengupta D and Chattopadhyay A (2015) Molecular dynamics simulations of GPCR-cholesterol interaction: An emerging paradigm Biochim Biophys Acta 1848 1775-82

Sengupta D, Prasanna X, Mohole M and Chattopadhyay A(2018) Exploring GPCR-lipid interactions by molecular dynamics simulations: Excitements, challenges, and the way forward J Phys Chem B 122 5727-5737

Senju Y, Kalimeri M, Koskela E V, Somerharju P, Zhao H, Vattulainen I and Lappalainen P (2017) Mechanistic principles underlying regulation of the actin cytoskeleton by phosphoinositides Proc Natl Acad Sci USA 114 E8977E8986

Senju Y and Lappalainen P (2019) Regulation of actin dynamics by $\mathrm{PI}(4,5) \mathrm{P} 2$ in cell migration and endocytosis Curr Opin Cell Biol 56713

Sheng M and Pak D T (2000) Ligand-gated ion channel interactions with cytoskeletal and signaling proteins Ann Rev Physiol 62 755-778

Sid T (2018) New opportunities for GPCR allosteric modulators Future Med Chem 10 707-710

Sriram K and Insel P A (2018) 'GPCRs as targets for approved drugs: How many targets and how many drugs Mol Pharm 117111062

Sun Y, Hu W, Yu X, Liu Z, Tarran R Ravid, K and Huang P (2016) Actinin-1 binds to the $\mathrm{C}$-terminus of $\mathrm{A} 2 \mathrm{~B}$ adenosine receptor (A2BAR) and enhances A2BAR cell-surface expression Biochem J 473 2179-2186

SuzukiK, Ritchie K, Kajikawa E, Fujiwara T and Kusumi A(2005) Rapid hop diffusion of a G-protein-coupled receptor in the plasma membrane as revealed by single-molecule techniques Biophys $J \mathbf{8 8} 3659-3680$

Tanaka K, Takeda S, Mitsuoka K, Oda T, Kimura-Sakiyama C, Maeda Y and Narita A (2018) Structural basis for cofilin binding and actin filament disassembly Nature 91860

Thal D, Vuckovic, Z, Draper-Joyce, C, Liang, Y L, Glukhova A, Christopoulos A and Sexton P (2018) Recent advances in the determination of $\mathrm{g}$ protein-coupled receptor structures Curr Opin Struct Biol 51 28-34

Tirupula K C, Ithychanda S S, Mohan M L, Naga Prasad S V, Qin J and Karnik S S (2015) G protein-coupled receptors directly bind filamin A with high affinity and promote filamin phosphorylation Biochemistry 54 6673-6683

Uribe Rand Jay D (2007) A review of actin binding proteins: New perspectives Mol Biol Rep 36 121-125

Varandas K C, Irannejad R and von Zastrow M (2016) Retromer Endosome Exit Domains Serve Multiple Trafficking Destinations and Regulate Local G Protein Activation by GPCRs Curr Biol 26 3129-3142

V'azquez-Victori G, Gonz'alez-Espinosa C, Espinosa-Riquer, Z P and Mac'ýas-Silva M. (2016) GPCRs and actincytoskeleton dynamics Methods in cell biology 132 165188

Venkatakrishnan A J, Deupi X, Lebon G, Tate C G, Schertler, G F and Babu M M (2013) Molecular signatures of G-proteincoupled receptors Nature 494 185-194

von der Ecken J, Mller M, Lehman W, Manstein D J, Penczek P A and Raunser S (2014) Structure of the F-actintropomyosin complex Nature 519 114-121

von der Ecken V and Heissler S M (2016) Cryo-EM structure of a human cytoplasmic actomyosin complex at near-atomic resolution Nature $\mathbf{5 3 4}$ 724-728

Weis W I and Kobilka B K (2018) The molecular basis of G protein-coupled receptor activation Ann Rev Biochem $\mathbf{8 7}$ 897-919

Winder S J and Ayscough K R (2005) Actin-binding proteins $J$ Cell Sci 118 651-654

Wirth A, Holst K and Ponimaskin E (2017) How serotonin receptors regulate morphogenic signaling in neurons Prog Neurobiol 151 35-56

Wisler J W, Rockman H A and Lefkowitz R J (2018) Biased G protein-coupled receptor signaling: Changing the paradigm of drug discovery Circulation 137 2315-2317

Zhao J, Wu J and Veatch S L (2013)Adhesion stabilizes robust lipid heterogeneity insupercritical membranes at physiological temperature Biophys J 104 825-834. 